Address for Correspondence: Dr. Ludovico Abenavoli, M.D. Ph.D. Department of Health Sciences, University "Magna Græcia” Viale Europa, 88100, Catanzaro, Italy. Email: I.abenavoli@unicz.it

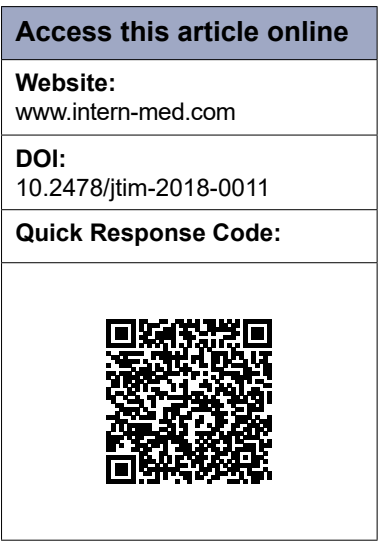

\title{
Resistin levels in non-alcoholic fatty liver disease pathogenesis
}

\author{
Carmela Colica ${ }^{1}$, Ludovico Abenavoli ${ }^{2}$ \\ 'CNR, IBFM UOS of Germaneto, University “Magna Graecia”, Catanzaro, Italy; \\ 2Department of Health Sciences, University “Magna Græcia”, Catanzaro, Italy
}

\section{TO THE EDITOR}

Non-alcoholic fatty liver disease (NAFLD) is an emerging public health issue, being not only a major cause of liver-related morbidity and mortality worldwide, but also an independent risk factor for chronic diseases. ${ }^{[1]}$ NAFLD is now considered the hepatic border of metabolic syndrome, supported by a high-calorie dietetic regimen, in the presence of a genetic profile. ${ }^{[2]}$ Several molecules produced by adipose tissue such as adiponectin, leptin, visfatin and resistin, and pro-inflammatory cytokines such as tumor necrosis factor- $\alpha$, and interleukins can be involved in the pathogenesis of NAFLD. ${ }^{[1,2]}$ In particular, resistin is a $10 \mathrm{kDa}$ dimeric protein secreted by adipose tissue and macrophages, the human gene codes for a 94 amino acids polypeptide. Resistin circulates in two distinct forms, the HMM hexamer (high-molecular-mass) that is the predominant state, and LMM complex (low-molecular-mass) the more bioactive peptide. It was reported in the studies on animal models that liver is a major target of resistin; in fact, it is involved in glucose and lipid metabolism, contributing to the development of insulin resistance and obesity. ${ }^{[2]}$

Resistin is also expressed in liver cells, where its production seems to increase with the progression of liver damage. In histologically confirmed NAFLD studies, the higher circulating levels of resistin were reported in patients with simple fat accumulation, or non-alcoholic steatohepatitis than in controls. ${ }^{[3]}$ However, literature also reported similar resistin levels in patients with simple steatosis compared to steatohepatitis. ${ }^{[4]}$
On this topic, we have previously published a study on the relationship between the serum levels of a selected set of adipokines included resistin, and NAFLD in overweight patients compared to the lean controls. ${ }^{[5]}$ We found no differences in the resistin serum levels in the patients with NAFLD compared to controls. However, we also demonstrated a negative correlation between resistin concentration and echographic stage of hepatic fat accumulation. These observations are similar to previous reports and suggest that the role of resistin in the pathogenesis of NAFLD can be limited. However, resistin could circulate in different molecular isoforms in peripheral human blood, and this might be the cause of the mistakes for the determination of its serum levels and for the comparison of the levels measured with different assay systems.

It seems reasonable to conclude that, in line with these conflicting data, the role and the contribution of resistin in NAFLD development and progression should be clarified in further studies.

\section{Conflict of Interest}

The authors declare no conflict of interest.

\section{REFERENCES}

1. Abenavoli L, Milic N, Di Renzo L, Preveden T, Medić-Stojanoska M, De Lorenzo A. Metabolic aspects of adult patients with nonalcoholic fatty liver disease. World J Gastroenterol 2016; 22: 7006-16.

2. Abenavoli L, Peta V. Role of adipokines and cytokines in non-alcoholic fatty liver disease. Rev Recent Clin Trials 2014; 9: 134-40.

3. Adolph TE, Grander C, Grabherr F, Tilg H. Adipokines and Non-Alcoholic Fatty Liver Disease: Multiple Interactions. Int J Mol Sci 2017;18: pii: E1649. 
Colica and Abenavoli: Resistin and non-alcoholic fatty liver disease

4. Shen C, Zhao CY, Wang W, Wang YD, Sun H, Cao W, et al. The relationship between hepatic resistin overexpression and inflammation in patients with nonalcoholic steatohepatitis. BMC Gastroenterol 2014; 14: 39.
5. Abenavoli L, Luigiano C, Guzzi PH, Milic N, Morace C, Stelitano L, et al. Serum adipokine levels in overweight patients and their relationship with non-alcoholic fatty liver disease. Panminerva Med 2014; 56: 189-93.

How to cite this article: Colica C, Abenavoli L. Resistin levels in nonalcoholic fatty liver disease pathogenesis. J TransI Intern Med 2018; 6: $52-3$. 